\title{
Mean-Variance Portfolio Optimization with State-Dependent Risk Aversion
}

Björk, Tomas; Murgoci, Agatha; Zhou, Xun Yu

\author{
Document Version \\ Final published version \\ Published in: \\ Mathematical Finance \\ DOI: \\ 10.1111/j.1467-9965.2011.00515.x \\ Publication date: \\ 2014 \\ License \\ CC BY-NC-ND
}

Citation for published version (APA):

Björk, T., Murgoci, A., \& Zhou, X. Y. (2014). Mean-Variance Portfolio Optimization with State-Dependent Risk Aversion. Mathematical Finance, 24(1), 1-24. https://doi.org/10.1111/j.1467-9965.2011.00515.x

Link to publication in CBS Research Portal

\section{General rights}

Copyright and moral rights for the publications made accessible in the public portal are retained by the authors and/or other copyright owners and it is a condition of accessing publications that users recognise and abide by the legal requirements associated with these rights.

\section{Take down policy}

If you believe that this document breaches copyright please contact us (research.lib@cbs.dk) providing details, and we will remove access to the work immediately and investigate your claim.

Download date: 26. Apr. 2023

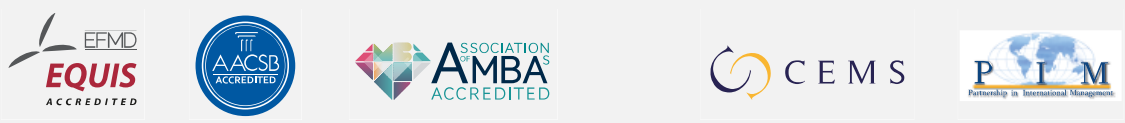


This is the accepted version of the following article: Björk, T., Murgoci, A. and Zhou, X. Y. (2014), MEAN-VARIANCE PORTFOLIO OPTIMIZATION WITH STATE-DEPENDENT RISK AVERSION. Mathematical Finance, 24: 1-24., which has been published in final form at doi: http://dx.doi.org/10.1111/j.1467-9965.2011.00515.x

\title{
Mean-Variance Portfolio Optimization with State Dependent Risk Aversion*
}

\author{
Tomas Björk ${ }^{\dagger} \quad$ Agatha Murgoci ${ }^{\ddagger} \quad$ Xun Yu Zhou $§$
}

July 8, 2011

First version October 2009

\begin{abstract}
The objective of this paper is to study the mean-variance portfolio optimization in continuous time. Since this problem is time inconsistent we attack it by placing the problem within a game theoretic framework and look for subgame perfect Nash equilibrium strategies. This particular problem has already been studied in [2] where the authors assumed a constant risk aversion parameter. This assumption leads to an equilibrium control where the dollar amount invested in the risky asset is independent of current wealth, and we argue that this result is unrealistic from an economic point of view. In order to have a more realistic model we instead study the case when the risk aversion depends dynamically on current wealth. This is a substantially more complicated problem than the one with constant risk aversion but, using the general theory of time inconsistent control developed in [4], we provide a fairly detailed analysis on the general case. In particular, when the risk aversion is inversely proportional to wealth, we provide an analytical solution where the equilibrium dollar amount invested in the risky asset is proportional to current wealth. The equilibrium for this model thus appears more reasonable than the one for the model with constant risk aversion.
\end{abstract}

Key words: Mean-variance, time inconsistency, time inconsistent control, dynamic programming, stochastic control, Hamilton-Jacobi-Bellman equation

${ }^{*}$ The authors are greatly indebted to Ivar Ekeland, Ali Lazrak, Traian Pirvu, and Suleyman Basak for very helpful discussions. We are also very grateful to two anonymous referees for a number of comments, which have improved the paper considerably.

$\dagger$ Department of Finance, Stockholm School of Economics, Box 6501, SE-113 83 Stockholm, SWEDEN. E-mail: tomas.bjork@hhs.se

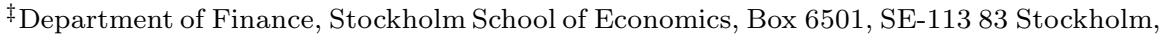
SWEDEN. E-mail: agatha.murgoci@hhs.se

§Mathematical Institute, University of Oxford, 24-29 St Giles', Oxford OX1 3LB, UK, and Deptartment of Systems Engineering \& Engineering Management, The Chinese University of Hong Kong, Shatin, Hong Kong. E-mail: zhouxy@maths.ox.ac.uk 


\section{Introduction}

Mean-variance (MV) analysis for optimal asset allocation is one of the classical results of financial economics. After the original publication in [14], a vast number of papers have been published on this topic. Most of these papers deal with the single period case, and there is a very good reason for this: It is very easy to see that an MV optimal portfolio problem in a multi period framework is time inconsistent in the sense that the Bellman Optimality Principle does not hold. As a consequence, dynamic programming cannot be easily applied, and it is in fact not at all clear what one should mean by the term "optimal".

In the literature there are two basic ways of handling (various forms of) time inconsistency in optimal control problems. One possibility is to study the pre-committed problem, where "optimal" is interpreted as "optimal from the point of view of time zero". Kydland and Prescott [10] indeed argue that a precommitted strategy may be economically meaningful in certain circumstances. In the context of MV portfolio choice, [17] is probably the earliest paper that studies a pre-committed MV model in a continuous-time setting (although he considers only one single stock with a constant risk-free rate), followed by [1]. In a discrete-time setting, [11] developed an embedding technique to change the originally time-inconsistent MV problem into a stochastic LQ control problem. This technique was extended in [21], along with an indefinite stochastic linearquadratic control approach, to the continuous-time case. Further extensions and improvements are carried out in, among many others, [13], [12], [3], and [20]. Markowitz's problem with transaction cost is recently solved in [5]. Note that in all these works only pre-committed strategies have been derived.

Another possibility is to take the time inconsistency more seriously and study the problem within a game theoretic framework. This is in fact the approach of the present paper. One possible interpretation of the time inconsistency is that our preferences change in a temporally inconsistent way as time goes by, and we can thus view the MV problem as a game, where the players are the future incarnations of our own preferences. We then look for a subgame perfect Nash equilibrium point for this game.

The game theoretic approach to addressing general time inconsistency via Nash equilibrium points has a long history starting with [18] where a deterministic Ramsey problem is studied. Further work along this line in continuous and discrete time is provided in [8], [9], [15], [16], and [19].

Recently there has been renewed interest in these problems. In the interesting papers [6] and [7], the authors consider optimal consumption and investment under hyperbolic discounting in deterministic and stochastic models from the above game theoretic point of view. To our knowledge, these papers were the first to provide a precise definition of the game theoretic equilibrium concept in continuous time.

In the particular case of MV analysis, the game theoretic approach to time inconsistency was first studied (in discrete and continuous time) in [2], where the authors undertake a deep study of the problem within a Wiener driven framework. The case of multiple assets, as well as the case of a hidden Markov 
process driving the parameters of the asset price dynamics are also treated. The authors derive an extension of the Hamilton-Jacobi-Bellman equation and manages, by a number of very clever ideas, to solve this equation explicitly for the basic problem, and also for the above mentioned extensions. The methodology of [2] is, among other things, to use a "total variance formula", which partially extends the standard iterated expectations formula. This works very nicely in the MV case, but drawback of this particular approach is that it seems quite hard to extend the results to other objective functions than MV.

The first paper to treat the game theoretic approach to time inconsistency in more general terms was [4] where the authors consider a fairly general class of (time inconsistent) objective functions and a very general controlled Markov process. Within this framework the authors derive an extension of the standard dynamic programming equation, to a system of equations (which in the diffusion case is a system of non linear PDEs). The framework of [4] is general enough to include many previously known models of time inconsistency. In particular, [4] reproduces the result of [2] for the MV problem in a Black-Scholes market.

Going back to the MV portfolio optimization, there is a non trivial problem connected with the solution presented in [2]. In the problem formulation of [2], the MV objective function at time $t$, given current wealth $X_{t}=x$, is given by

$$
E_{t, x}\left[X_{T}\right]-\frac{\gamma}{2} \operatorname{Var}_{t, x}\left[X_{T}\right],
$$

where $X_{T}$ is the wealth at the end of the time period, and where $\gamma$ is a given constant representing the risk aversion of the agent. For such a model it turns out (see [2]) that, at time $t$ and when total wealth is $x$, the optimal dollar amount $u(t, x)$ invested in the risky asset is of the form

$$
u(t, x)=h(t)
$$

where $h$ is a deterministic function of time. In particular this implies that the dollar amount invested in the risky asset does not depend on current wealth $x$.

In our opinion this result is economically unreasonable, since it implies that you will invest the same number of dollars in the stock if your wealth is 100 dollars as you would if your wealth is $100,000,000$ dollars. See Section ?? for a more detailed discussion.

The deeper reason for this anomaly is the fact that the risk aversion parameter $\gamma$ is assumed to be a constant, which is clearly unreasonable. A person's risk preference certainly depends on how wealthy he is; and hence the obvious implication is that we should explicitly allow $\gamma$ to depend on current wealth.

The main goal of the present paper is precisely to study MV problems with a state dependent risk aversion. More explicitly we consider an objective function of the form

$$
E_{t, x}\left[X_{T}\right]-\frac{\gamma(x)}{2} \operatorname{Var}_{t, x}\left[X_{T}\right]
$$

where $\gamma$ is a deterministic function of present wealth $x$. This type of problem cannot easily be treated within the framework of [2], but it is a simple special case of the theory developed in [4]. 
The structure and main result of the present paper are as follows. In Section 2 we present our formal model. We discuss the time inconsistency of the mean variance problem and we place that problem within the general framework of [4]. The game theoretic problem is presented, both in informal and in mathematically precise terms, and from [4] we cite the general theoretical results that we need for our analysis.

In Section 3 we give a brief recapitulation of the MV problem with constant $\gamma$ studied in [2], and we use our general theory to derive the solution. In Section 4 we study the MV problem with state dependent risk aversion using the theory developed in [4]. We start by deriving a surprisingly explicit solution for the case of a general risk aversion $\gamma(x)$. We then specialize to the economically natural case of $\gamma(x)=\gamma / x$ and for this case we obtain an analytic solution. More precisely, we show that the optimal dollar amount $\hat{\mathbf{u}}_{t}$ to invest in the risky asset at time $t$ is given by

$$
\hat{\mathbf{u}}_{t}=c(t) x
$$

where the deterministic function $c$ solves an integral equation. This is the main result of the paper, and it shows that with the proper specification of the risk aversion $\gamma(x)$, the optimal solution is indeed economically reasonable.

We finish the paper by proving that the integral equation for $c$ admits a unique solution, and we also provide a numerical algorithm for computing $c$. The algorithm is implemented for some natural parameter combinations and we present graphs for illustrative purposes.

\section{The basic framework}

In this section we formulate the problem under consideration.

\subsection{The model}

Our basic setup is a standard Black-Scholes model for a risky stock with GBM price dynamics and a bank account with constant risk free short rate $r$. Denoting the stock price by $S$ and the bank account by $B$, the dynamics are as follows under the objective probability measure $P$.

$$
\begin{aligned}
d S_{t} & =\alpha S_{t} d t+\sigma S_{t} d W_{t}, \\
d B_{t} & =r B_{t} d t .
\end{aligned}
$$

Here $W$ is a standard $P$-Wiener process, and the constants $\alpha, \sigma$, and $r$ are assumed to be known. In the analysis below, we will study self-financing portfolios (with zero consumption), based on $S$ and $B$. Denoting the dollar value invested in the risky asset at time $t$ by $u_{t}$, the value process $X$ of the portfolio is easily seen to have dynamics given by

$$
d X_{t}=\left[r X_{t}+(\alpha-r) u_{t}\right] d t+\sigma u_{t} d W_{t} .
$$




\subsection{The basic problem}

Loosely speaking, the problem to be solved is to maximize utility of final wealth, where the utility is of MV form, i.e. we want to maximize the expression

$$
E\left[X_{T}\right]-\frac{\gamma}{2} \operatorname{Var}\left[X_{T}\right],
$$

where $\gamma$ is a pre-specified risk aversion parameter.

A disturbing property of this optimization problem is that it is time inconsistent in the sense that the Bellman optimality principle does not hold. For a more detailed discussion see $[2,4]$.

The lack of time consistency leads to conceptual as well as computational problems. From a conceptual point of view, it is no longer clear what we mean by the word "optimal", since a control strategy which is optimal for one choice of starting point in time will generically not be optimal at a later point in time. On the other hand, even with certain precise definition of optimality, we also have a computational problem, since DynP is no longer available.

There are at least two approaches to the conceptual problem.

- We fix one initial point, like for example $\left(0, x_{0}\right)$, and then try to find the control law $\hat{\mathbf{u}}$ which maximizes $E\left[X_{T}\right]-\frac{\gamma}{2} \operatorname{Var}\left[X_{T}\right]$ where $X_{0}=x_{0}$. We then simply disregard the fact that at a later points in time the control law $\hat{\mathbf{u}}$ will not be optimal. Such a control is known as pre-commitment.

- We take the time inconsistency seriously and formulate the problem in game theoretic terms.

In this paper we will use the game theoretic formulation.

\subsection{The game theoretic formulation}

In order to formulate the game theoretic version of our problem, we will follow [4], where the reader may find full proofs of all cited results. To see the structure of the problem a bit more clearly we will consider a more general and abstract problem than the MV problem above. This will also allow us to treat the case with a state dependent $\gamma$ below.

We thus consider a controlled $n$-dimensional SDE of the form

$$
d X_{t}=\mu\left(t, X_{t}, u_{t}\right) d t+\sigma\left(t, X_{t}, u_{t}\right) d W_{t}
$$

where $W$ is $P$-Wiener. The adapted control $u$ is restricted to takes values in $\mathcal{U} \subseteq R^{k}$. We restrict ourselves to feedback control laws, i.e. the controls are of the form $u_{t}=\mathbf{u}\left(t, X_{t}\right)$ where the control law $\mathbf{u}: R_{+} \times R^{n} \rightarrow R^{k}$ is a deterministic function of the two variables $t$ and $x$. The solution of the SDE above, using the control law $\mathbf{u}$ will be denoted by $X^{\mathbf{u}}$.

For a fixed initial point $(t, x)$ we consider a functional of the form

$$
J(t, x, \mathbf{u})=E_{t, x}\left[F\left(x, X_{T}^{\mathbf{u}}\right)\right]+G\left(x, E_{t, x}\left[X_{T}^{\mathbf{u}}\right]\right),
$$


where $F(x, y)$ and $G(x, y)$ are given deterministic functions. The objective is to maximize $J(t, x, \mathbf{u})$ for each $(t, x)$. We see that with the choice of the functional above, time inconsistency enters at several different points. Firstly we have the appearance of the present state $x=X_{t}$ in the functions $F$ and $G$, and this leads to time inconsistency since it implies that, as the state process $X$ changes, our preferences change accordingly. Secondly, in the term $G\left(x, E_{t, x}\left[X_{T}^{\mathbf{u}}\right]\right)$ we have, even without the presence of $x$, a nonlinear function $G$ acting on the conditional expectation, and this again leads to time inconsistency.

In order to define our game we now make the interpretation that at every point in time $t$ we have one player - player number $t$ - which we will denote by $P_{t}$. Conceptually we may think of this as a game with an infinite number of distinct players - one at each point in time. Alternatively you may think of the game as a game with one "real" player namely yourself, and $P_{t}$ as the incarnation of your own preferences at time $t$. Given a control law $\mathbf{u}$, the reward function for $P_{t}$ is given by $J(t, x, \mathbf{u})$, where $J$ obviously only depends on the control law $\mathbf{u}$ restricted to the time interval $[t, T]$.

We can now loosely define the concept of a "subgame perfect Nash equilibrium point" of the game. This is a control law $\hat{\mathbf{u}}$ satisfying the following:

- Choose an arbitrary point $t$ in time.

- Suppose that every player $s$, for all $s>t$, will use the strategy $\hat{\mathbf{u}}(s, \cdot)$.

- Then the optimal choice for player $t$, given the objective functional $J$ defined above, is that he/she also uses the strategy $\hat{\mathbf{u}}(t, \cdot)$.

Based on this we now give the formal definition of an equilibrium control.

Definition 2.1 Given a control law $\hat{\mathbf{u}}$, construct a control law $\mathbf{u}_{h}$ by

$$
\mathbf{u}_{h}(s, y)=\left\{\begin{array}{cl}
u, & \text { for } t \leq s<t+h, \quad y \in R^{n} \\
\hat{\mathbf{u}}(s, y), & \text { for } t+h \leq s \leq T, \quad y \in R^{n}
\end{array}\right.
$$

where $u \in R^{k}, h>0$, and $(t, x) \in[0, T] \times R^{n}$ are arbitrarily chosen. If

$$
\liminf _{h \rightarrow 0} \frac{J(t, x, \hat{\mathbf{u}})-J\left(t, x, \mathbf{u}_{h}\right)}{h} \geq 0,
$$

for all $u \in R^{k}$ and $(t, x) \in[0, T] \times R^{n}$, we say that $\hat{\mathbf{u}}$ is an equilibrium control law. The equilibrium value function $V$ is defined by

$$
V(t, x)=J(t, x, \hat{\mathbf{u}}) .
$$

We note that in the case of a standard time consistent setting, the definition of an equilibrium will coincide with the definition of an optimal strategy and the equilibrium value function will be the usual optimal value function.

As a special case of the results on [4] we can now define the appropriate extension of the standard Hamilton-Jacobi-Bellman (HJB) equation. The operator $\mathbf{A}^{u}$ below is the usual controlled infinitesimal operator defined by

$$
\mathbf{A}^{u}=\frac{\partial}{\partial t}+\mu(t, x, u) \frac{\partial}{\partial x}+\frac{1}{2} \sigma^{2}(t, x, u) \frac{\partial^{2}}{\partial x^{2}} .
$$


Definition 2.2 The extended HJB system of equations for the Nash equilibrium problem is defined as follows.

$$
\begin{aligned}
\sup _{u \in \mathcal{U}}\left\{\left(\mathbf{A}^{u} V\right)(t, x)-\left(\mathbf{A}^{u} f\right)(t, x, x)+\left(\mathbf{A}^{u} f^{x}\right)(t, x)\right. & \\
\left.-\mathbf{A}^{u}(G \diamond g)(t, x)+\left(\mathbf{H}^{u} g\right)(t, x)\right\} & =0, \quad 0 \leq t \leq T \\
\mathbf{A}^{\hat{\mathbf{u}}} f^{y}(t, x) & =0, \quad 0 \leq t \leq T, \\
\mathbf{A}^{\hat{\mathbf{u}}} g(t, x) & =0, \quad 0 \leq t \leq T, \\
V(T, x) & =F(x, x)+G(x, x), \\
f(T, x, y) & =F(y, x), \\
g(T, x) & =x .
\end{aligned}
$$

Here $\hat{\mathbf{u}}$ is the control law which realizes the supremum in the first equation, and $f^{y}, G \diamond g$, and $\mathbf{H} g$ are defined by

$$
\begin{aligned}
f^{y}(t, x) & =f(t, x, y), \\
(G \diamond g)(t, x) & =G(x, g(t, x)), \\
\mathbf{H}^{u} g(t, x) & =G_{y}(x, g(t, x)) \cdot \mathbf{A}^{u} g(t, x), \\
G_{y}(x, y) & =\frac{\partial G}{\partial y}(x, y) .
\end{aligned}
$$

We now have some comments on the extended HJB system.

- The first point to notice is that we have a system of deterministic recursion equations for the simultaneous determination of $V(t, x), f(t, x, y)$ and $g(t, x)$.

- In the case when $F(x, y)$ does not depend upon $x$, and there is no $G$ term, the problem trivializes to a standard time consistent problem. The terms $\left(\mathbf{A}^{u} f\right)(t, x, x)+\left(\mathbf{A}^{u} f^{x}\right)(t, x)$ in the $V$-equation cancel, and the system reduces to the standard HJB equation

$$
\begin{aligned}
\left(\mathbf{A}^{u} V\right)(t, x) & =0, \\
V(T, x) & =F(x) .
\end{aligned}
$$

- We have the probabilistic interpretations

$$
\begin{aligned}
f(t, x, y) & =E_{t, x}\left[F\left(y, X_{T}^{\hat{\mathbf{u}}}\right)\right], \\
g(t, x) & =E_{t, x}\left[X_{T}^{\hat{\mathbf{u}}}\right] .
\end{aligned}
$$

- The equations for $g$ and $f^{y}$ state that the processes $g\left(t, X_{t}^{\hat{u}}\right)$ and $f^{y}\left(t, X_{t}^{\hat{\mathbf{u}}}\right)$ are martingales.

We finish this section by citing the Verification Theorem from [4]. This shows that if you mange to solve the (deterministic) extended HJB system, then you have in fact solved your game. 
Theorem 2.1 (Verification Theorem) Assume that $(V, f, g)$ is a solution of the extended system in Definition 2.2, and that the control law $\hat{\mathbf{u}}$ realizes the supremum in the equation. Then $\hat{\mathbf{u}}$ is an equilibrium control law, and $V$ is the corresponding value function. Furthermore, $f$ and $g$ can be interpreted according to (1)-(2).

We will now use this result to analyze two versions of the MV portfolio optimization problem.

\section{Mean-variance with constant risk aversion}

In this section we will review the result of [4] based on total variance formula (and reproduced in [4] based on the extended HJB equation) for the simplest case of MV portfolio optimization with constant risk aversion. Then we will discuss it from an economic perspective and we will find that it is not economically reasonable. In Section 4 we will therefore extend the "naive" MV problem to a more realistic one where $\gamma$ is allowed to depend on current wealth $x$.

The problem is already presented in Section 2.2. To recall, the state dynamics are given by

$$
d X_{t}=\left[r X_{t}+(\alpha-r) u_{t}\right] d t+\sigma u_{t} d W_{t}
$$

and the reward function to player number $t$ is given by

$$
J(t, x, \mathbf{u})=E_{t, x}\left[X_{T}^{\mathbf{u}}\right]-\frac{\gamma}{2} \operatorname{Var}_{t, x}\left[X_{T}^{\mathbf{u}}\right] .
$$

We can re-write our objective functional as

$$
J(t, x, \mathbf{u})=E_{t, x}\left[F\left(X_{T}^{\mathbf{u}}\right)\right]-G\left(E_{t, x}\left[X_{T}^{\mathbf{u}}\right]\right)
$$

where $F(x)=x-\frac{\gamma}{2} x^{2}$ and $G(x)=\frac{\gamma}{2} x^{2}$.

Proposition 3.1 (Basak-Chabakauri [2]) For the model above we have the following results.

- The optimal amount of money invested in a stock is given by

$$
\hat{\mathbf{u}}(t, x)=\frac{1}{\gamma} \frac{\alpha-r}{\sigma^{2}} e^{-r(T-t)} .
$$

- The equilibrium value function is given by

$$
V(t, x)=e^{r(T-t)} x+\frac{1}{2 \gamma} \frac{(\alpha-r)^{2}}{\sigma^{2}}(T-t) .
$$

- The expected value of the optimal portfolio is given by

$$
E_{t, x}\left[X_{T}^{\hat{\mathbf{u}}}\right]=e^{r(T-t)} x+\frac{1}{\gamma} \frac{(\alpha-r)^{2}}{\sigma^{2}}(T-t) .
$$


Let us now discuss the equilibrium solution above from an economic point of view, and the main question is if the result makes economic sense. Our claim is in fact that the result does not make economic sense, and to see this we recall that the equilibrium portfolio is given by

$$
\hat{\mathbf{u}}(t, x)=\frac{1}{\gamma} \frac{\alpha-r}{\sigma^{2}} e^{-r(T-t)} .
$$

We now recall that the interpretation of the control $u$ is the dollar amount that is invested in the risky asset. From the expression for $\hat{\mathbf{u}}$ we thus see that the dollar amount invested in the risky asset at time $t$ is independent of the current wealth $x$. This is, in our view, economically unrealistic. The result implies that you will invest the same number of dollars in the stock if your wealth is 100 dollars as you would if your wealth is 100,000,000 dollars. Stated this way, it is clear that the equilibrium portfolio is economically unreasonable. We hasten to add that there is nothing surprising about the result - given the experience from the one-period mean variance model this particular result is quite expected.

There is, however, a big conceptual difference between a one-period model and our multi-period model. In the one-period model one can argue that although the independence of initial wealth seems unreasonable at first sight, we can save the situation by noting that the risk aversion parameter $\gamma$ should depend on the initial wealth. The obvious intuition is of course that $\gamma$ should decrease with increasing wealth, so the person with initial wealth of 100 should have a much higher value of $\gamma$ than a fund with 100,000,000 initial capital.

This idea is, in our view, perfectly reasonable in a one-period setting. It is also reasonable for the case of pre-committment, since then the perspective is clearly taken at time $t=0$.

In our multi-period setting, however, and with our game theoretic formulation, we do have a problem, since in this setting there is no particular importance attached to the wealth at time $t=0$. Instead, all points in time are of equal importance. We can of course choose our $\gamma$ such that it reflects the risk version corresponding to the initial wealth $x_{0}$. As time goes by, however, our wealth will change so when we come to time $t$ the risk aversion coefficient $\gamma$ which was appropriate for the initial wealth $x_{0}$ is no longer appropriate for the current wealth $X_{t}$.

The natural conclusion of this discussion is, in our opinion, that we should explicitly allow the risk aversion parameter $\gamma$ to be a function $\gamma(x)$ of current wealth $x$. In the next section we will analyze this case.

\section{Mean-variance with state dependent risk aver- sion}

From the discussion in the previous section it is clear that we should extend the simple MV problem treated in [2] to the more realistic case when the reward 
functional is given by

$$
J(t, x, \mathbf{u})=E_{t, x}\left[X_{T}^{\mathbf{u}}\right]-\frac{\gamma(x)}{2} \operatorname{Var}_{t, x}\left[X_{T}^{\mathbf{u}}\right]
$$

where $\gamma$ is a deterministic function of $x$. We now have two problems.

- Given a specific choice of $\gamma(x)$, how do we compute the equilibrium strategy.

- Is there a particularly natural choice of the risk aversion function $\gamma(x)$ ?

To start with the first question, it is clear that the problem is perfectly fitted to be attacked by the extended HJB system in Definition 2.2. In fact, the reward functional can be written as

$$
J(t, x, \mathbf{u})=E_{t, x}\left[F\left(x, X_{T}^{\mathbf{u}}\right)\right]-G\left(x, E_{t, x}\left[X_{T}^{\mathbf{u}}\right]\right)
$$

with

$$
\begin{aligned}
& F(x, y)=y-\frac{\gamma(x)}{2} y^{2}, \\
& G(x, y)=\frac{\gamma(x)}{2} y^{2} .
\end{aligned}
$$

It is thus clear that we are within the general framework of [4].

\subsection{A natural choice of $\gamma(x)$}

We now turn to the second question above, namely that of having a "natural" choice for $\gamma(x)$. There are in fact two natural arguments, both leading to the same choice of $\gamma(x)$.

1. One way of finding a natural candidate of $\gamma$ is to perform a dimension analysis. In the reward function

$$
J(t, x, \mathbf{u})=E_{t, x}\left[X_{T}^{\mathbf{u}}\right]-\frac{\gamma(x)}{2} \operatorname{Var}_{t, x}\left[X_{T}^{\mathbf{u}}\right]
$$

we see that the first term $E_{t, x}\left[X_{T}^{\mathbf{u}}\right]$ has the dimension (dollar). The term $\operatorname{Var}_{t, x}\left[X_{T}^{\mathbf{u}}\right]$ has the dimension $(\text { dollar })^{2}$, so in order to have a reward function measured in dollars we have to choose $\gamma$ in such a way that $\gamma(x)$ has the dimension $(\text { dollar })^{-1}$. The most obvious way to accomplish this is of course to specify $\gamma$ as

$$
\gamma(x)=\frac{\gamma}{x}
$$

where, with a slight abuse of notation, the occurrence of $\gamma$ in the right hand side denotes a constant. 
2. In the original single-period mean variance analysis, the mean variance utility function (with constant $\gamma$ ) is in fact not applied to the wealth itself, but rather to the return rate. More precisely this means that the objective function is given by

$$
J(t, x, \mathbf{u})=E_{t, x}\left[\frac{X_{T}^{\mathbf{u}}}{x}\right]-\frac{\gamma}{2} \operatorname{Var}_{t, x}\left[\frac{X_{T}^{\mathbf{u}}}{x}\right]
$$

and we can write this as

$$
J(t, x, \mathbf{u})=\frac{1}{x}\left\{E_{t, x}\left[X_{T}^{\mathbf{u}}\right]-\frac{\gamma}{2 x} \operatorname{Var}_{t, x}\left[X_{T}^{\mathbf{u}}\right]\right\}
$$

Since $x>0$, it is clear that this objective functional will lead to the same equilibrium control $\hat{\mathbf{u}}$ as the objective functional

$$
J(t, x, \mathbf{u})=E_{t, x}\left[X_{T}^{\mathbf{u}}\right]-\frac{\gamma(x)}{2} \operatorname{Var}_{t, x}\left[X_{T}^{\mathbf{u}}\right] .
$$

with

$$
\gamma(x)=\frac{\gamma}{x}
$$

In the next subsection we will analyze the case of a general choice of $\gamma(x)$ and obtain a fairly explicit result. In Section 4.3 we specialize to the case when $\gamma(x)=\gamma / x$, and for this case we obtain a more explicit solution, which we will argue is also reasonable from an economic point of view.

\subsection{The case of a general $\gamma(x)$}

Applying the general theory from Section 2.3 to the objective functional

$$
J(t, x, \mathbf{u})=E_{t, x}\left[X_{T}^{\mathbf{u}}\right]-\frac{\gamma(x)}{2} \operatorname{Var}_{t, x}\left[X_{T}^{\mathbf{u}}\right]
$$

gives us, after a large number of elementary calculations, the following result (see Appendix A for details). We use the notation $\beta=\alpha-r$.

Proposition 4.1 The extended HJB system takes the following form:

$$
\begin{aligned}
V_{t}+\sup _{u \in R}\left\{(r x+\beta u)\left[V_{x}-f_{y}-\frac{\gamma^{\prime}(x)}{2} g^{2}\right]\right. & \\
\left.+\frac{1}{2} \sigma^{2} u^{2}\left[V_{x x}-f_{y y}-2 f_{x y}-\frac{\gamma^{\prime \prime}(x)}{2} g^{2}-2 \gamma^{\prime}(x) g g_{x}-\gamma(x) g_{x}^{2}\right]\right\} & =0, \\
f_{t}(t, x, y)+(r x+\beta \hat{u}) f_{x}(t, x, y)+\frac{1}{2} \sigma^{2} \hat{u}^{2} f_{x x}(t, x, y) & =0, \\
g_{t}(t, x)+(r x+\beta \hat{u}) g_{x}(t, x)+\frac{1}{2} \sigma^{2} \hat{u}^{2} g_{x x}(t, x) & =0 .
\end{aligned}
$$

Remark 4.1 Note that, in (3), the partial derivatives of $f$ and $g$ should be evaluated at $(t, x, x)$ and $(t, x)$ respectively. 
The HJB system above can in fact be simplified. We recall the probabilistic interpretations

$$
\begin{aligned}
V(t, x) & =E_{t, x}\left[X_{T}^{\hat{\mathbf{u}}}\right]-\frac{\gamma(x)}{2} \operatorname{Var}_{t, x}\left[X_{T}^{\hat{\mathbf{u}}}\right], \\
f(t, x, y) & =E_{t, x}\left[X_{T}^{\hat{\mathbf{u}}}\right]-\frac{\gamma(y)}{2} E_{t, x}\left[\left(X_{T}^{\hat{\mathbf{u}}}\right)^{2}\right] \\
g(t, x) & =E_{t, x}\left[X_{T}^{\hat{\mathbf{u}}}\right] .
\end{aligned}
$$

From these it follows that

$$
V(t, x)=f(t, x, x)+\frac{\gamma(x)}{2} g^{2}(t, x) .
$$

We thus have, always with $f$ and its derivatives evaluated at $(t, x, x)$,

$$
\begin{aligned}
V_{t} & =f_{t}+\gamma g g_{t}, \\
V_{x} & =f_{x}+f_{y}+\frac{\gamma^{\prime}}{2} g^{2}+\gamma g g_{x}, \\
V_{x x} & =f_{x x}+f_{y y}+2 f_{x y}+\frac{\gamma^{\prime \prime}}{2} g^{2}+2 \gamma^{\prime} g g_{x}+\gamma g_{x}^{2}+\gamma g g_{x x} .
\end{aligned}
$$

Using these expressions, equation (3) takes the form

$$
f_{t}+\gamma g g_{t}+\sup _{u \in R}\left\{(r x+\beta u)\left[f_{x}+\gamma g g_{x}\right]+\frac{1}{2} \sigma^{2} u^{2}\left[f_{x x}+\gamma g g_{x x}\right]\right\}=0
$$

and the optimal $u$ is easily obtained as

$$
\hat{u}(t, x)=-\frac{\beta}{\sigma^{2}} \frac{f_{x}(t, x, x)+\gamma(x) g(t, x) g_{x}(t, x)}{f_{x x}(t, x, x)+\gamma(x) g(t, x) g_{x x}(t, x)} .
$$

We collect our results

Lemma 4.1 With notation as above, the extended HJB system takes the form

$$
\begin{aligned}
f_{t}+\gamma g g_{t}+\sup _{u \in R}\left\{(r x+\beta u)\left[f_{x}+\gamma g g_{x}\right]+\frac{1}{2} \sigma^{2} u^{2}\left[f_{x x}+\gamma g g_{x x}\right]\right\} & =0, \\
f_{t}(t, x, y)+(r x+\beta \hat{u}) f_{x}(t, x, y)+\frac{1}{2} \sigma^{2} \hat{u}^{2} f_{x x}(t, x, y) & =0, \\
g_{t}(t, x)+(r x+\beta \hat{u}) g_{x}(t, x)+\frac{1}{2} \sigma^{2} \hat{u}^{2} g_{x x}(t, x) & =0,
\end{aligned}
$$

and the equilibrium control is given by

$$
\hat{u}(t, x)=-\frac{\beta}{\sigma^{2}} \frac{f_{x}(t, x, x)+\gamma(x) g(t, x) g_{x}(t, x)}{f_{x x}(t, x, x)+\gamma(x) g(t, x) g_{x x}(t, x)} .
$$


Since the extended HJB system above gives us three equations involving only two unknown functions $f$ and $g$, it now seems that we may have a potential problem with an over-determined system. This is, however, not the case. We recall that (4) is valid for all $x$ and $y$, so in particular we can set $y=x$. We then have the system

$$
\begin{aligned}
f_{t}+\gamma g g_{t}+(r x+\beta \hat{u})\left[f_{x}+\gamma g g_{x}\right]+\frac{1}{2} \sigma^{2} \hat{u}^{2}\left[f_{x x}+\gamma g g_{x x}\right] & =0, \\
f_{t}(t, x, x)+(r x+\beta \hat{u}) f_{x}(t, x, x)+\frac{1}{2} \sigma^{2} \hat{u}^{2} f_{x x}(t, x, x) & =0, \\
g_{t}(t, x)+(r x+\beta \hat{u}) g_{x}(t, x)+\frac{1}{2} \sigma^{2} \hat{u}^{2} g_{x x}(t, x) & =0 .
\end{aligned}
$$

This looks over-determined but by inspection we see that in fact

$$
(6)=(7)+\gamma g \cdot(8),
$$

so (6) is in fact implied by (7) and (8). We can thus summarize the results so far.

Proposition 4.2 The equilibrium control is given by

$$
\hat{u}(t, x)=-\frac{\beta}{\sigma^{2}} \frac{f_{x}(t, x, x)+\gamma(x) g(t, x) g_{x}(t, x)}{f_{x x}(t, x, x)+\gamma(x) g(t, x) g_{x x}(t, x)},
$$

where the functions $f$ and $g$ are determined by the system

$$
\begin{aligned}
f_{t}(t, x, y)+(r x+\beta \hat{u}) f_{x}(t, x, y)+\frac{1}{2} \sigma^{2} \hat{u}^{2} f_{x x}(t, x, y) & =0, \\
g_{t}(t, x)+(r x+\beta \hat{u}) g_{x}(t, x)+\frac{1}{2} \sigma^{2} \hat{u}^{2} g_{x x}(t, x) & =0
\end{aligned}
$$

with boundary conditions

$$
\begin{aligned}
f(T, x, y) & =x-\frac{\gamma(y)}{2} x^{2}, \\
g(T, x) & =x .
\end{aligned}
$$

The equilibrium value function $V$ is given by

$$
V(t, x)=f(t, x, x)+\frac{\gamma(x)}{2} g^{2}(t, x) .
$$

\subsection{The case $\gamma(x)=\frac{\gamma}{x}$}

We now move to the particularly interesting case when

$$
\gamma(x)=\frac{\gamma}{x} .
$$

Our main result is as follows. 
Proposition 4.3 For the case when $\gamma(x)=\frac{\gamma}{x}$, the equilibrium control is given by

$$
\hat{u}(t, x)=\frac{\beta}{\gamma \sigma^{2}} \frac{a(t)+\gamma\left[a^{2}(t)-b(t)\right]}{b(t)} x
$$

where $a$ and $b$ solves the ODE system

$$
\begin{aligned}
\dot{a}+\left(r+\frac{\beta^{2}}{\gamma \sigma^{2} b}\left(a+\gamma\left[a^{2}-b\right]\right)\right) a & =0, \\
a(T, x) & =1, \\
\dot{b}+\left\{2\left(r+\frac{\beta^{2}}{\gamma \sigma^{2} b}\left(a+\gamma\left[a^{2}-b\right]\right)\right)+\frac{\beta^{2}}{\gamma^{2} \sigma^{2} b^{2}}\left(a+\gamma\left[a^{2}-b\right]\right)^{2}\right\} b & =0, \\
b(T, x) & =1 .
\end{aligned}
$$

The equilibrium value function $V$ is given by

$$
V(t, x)=\left\{a(t)+\frac{\gamma}{2}\left[a^{2}(t)-b(t)\right]\right\} x .
$$

Proof. See Appendix B.

\subsection{An integral equation for $c$}

The ODE system in Proposition 4.3 is highly nonlinear, and it does not satisfy the usual Lipschitz and linear growth conditions guaranteeing global existence and uniqueness of a solution, so we have a problem. Instead of analyzing $a$ and $b$ as above, an alternative approach is to focus directly on the function $c$ in the Ansatz $\hat{u}(t, x)=c(t) x$ and to derive a single integral equation for $c$. Then we express $a$ and $b$ in terms of $c$.

Theorem 4.1 For the case $\gamma(x)=\frac{\gamma}{x}$, the equilibrium control is given by

$$
\hat{u}(t, x)=c(t) x
$$

where $c$ solves the integral equation

$$
c(t)=\frac{\beta}{\gamma \sigma^{2}}\left\{e^{-\int_{t}^{T}\left[r+\beta c(s)+\sigma^{2} c^{2}(s)\right] d s}+\gamma e^{-\int_{t}^{T} \sigma^{2}(s) c^{2}(s) d s}-\gamma\right\} .
$$

The equilibrium value function $V$ is given by

$$
V(t, x)=\left\{a(t)+\frac{\gamma}{2}\left[a^{2}(t)-b(t)\right]\right\} x
$$

where

$$
\begin{aligned}
& a(t)=e^{\int_{t}^{T}[r+\beta c(s)] d s}, \\
& b(t)=e^{2 \int_{t}^{T}\left[r+\beta c(s)+\frac{1}{2} \sigma^{2} c^{2}(s)\right] d s} .
\end{aligned}
$$


Proof. Under $\hat{u}(t, x)=c(t) x$ the wealth process $X$ is GBM:

$$
d X_{t}^{\hat{u}}=[r+\beta c(t)] X_{t}^{\hat{u}} d t+\sigma c(t) X_{t}^{\hat{u}} d W_{t} .
$$

A direct computation shows that

$$
\begin{aligned}
E_{t, x}\left[X_{T}^{\hat{u}}\right] & =x e^{\int_{t}^{T}[r+\beta c(s)] d s}, \\
E_{t, x}\left[\left(X_{T}^{\hat{u}}\right)^{2}\right] & =x^{2} e^{2 \int_{t}^{T}\left[r+\beta c(s)+\frac{1}{2} \sigma^{2} c^{2}(s)\right] d s} .
\end{aligned}
$$

Thus (14)-(15) follows from (26)-(27). From Proposition 4.3 we also have

$$
c(t)=\frac{\beta}{\gamma \sigma^{2}} \frac{a(t)+\gamma\left[a^{2}(t)-b(t)\right]}{b(t)},
$$

and a simple calculation, using (14)-(15), gives us (13).

The key question now is whether the integral equation (13) has a unique global solution. We assume from now on that $\beta:=\alpha-r>0$ (the case $\beta<0$ can be treated similarly). We also assume that $\gamma>0$ which is economically reasonable. We use the standard notation $C[0, T]$ to denote the space of continuous functions on $[0, T]$ endowed with supremum norm.

Theorem 4.2 The integral equation (13) admits a unique solution $c \in C[0, T]$.

Proof. We construct a sequence $c_{n} \in C[0, T]$ as follows

$$
\begin{aligned}
c_{0}(t) & \equiv 1 \\
c_{n}(t) & =\frac{\beta}{\gamma \sigma^{2}}\left[e^{-\int_{t}^{T}\left[r+\beta c_{n-1}(s)+\sigma^{2} c_{n-1}^{2}(s)\right] d s}+\gamma e^{-\int_{t}^{T} \sigma^{2} c_{n-1}^{2}(s) d s}-\gamma\right]
\end{aligned}
$$

$n=1,2, \cdots$. We will now show that this sequence converges in $C[0, T]$, and this will be done in three steps.

Step 1: Prove that $\left\{c_{n}\right\}$ is uniformly bounded in $C[0, T]$.

Noting that $\gamma>0$ we have

$$
c_{n}(t)=\frac{\beta}{\gamma \sigma^{2}} e^{-\int_{t}^{T} \sigma^{2} c_{n-1}^{2}(s) d s}\left\{e^{-\int_{t}^{T}\left[r+\beta c_{n-1}(s)\right] d s}+\gamma\right\}-\frac{\beta}{\sigma^{2}} \geq-\frac{\beta}{\sigma^{2}}
$$

for all $t \in[0, T]$. Using this we also obtain

$$
\begin{aligned}
c_{n}(t) & \leq \frac{\beta}{\gamma \sigma^{2}}\left[e^{-\int_{t}^{T} \beta c_{n-1}(s) d s}+\gamma-\gamma\right] \leq \frac{\beta}{\gamma \sigma^{2}} e^{-\int_{t}^{T} \beta \frac{\beta}{\sigma^{2}}} d s \\
& =\frac{\beta}{\gamma \sigma^{2}} e^{-\frac{\beta^{2}}{\sigma^{2}}(T-t)} \leq \frac{\beta}{\gamma \sigma^{2}} e^{\frac{\beta^{2}}{\sigma^{2}} T}, \quad \forall t, \quad n=1,2, \ldots
\end{aligned}
$$


We have thus proved that

$$
-\frac{\beta}{\sigma^{2}} \leq c_{n}(t) \leq \frac{\beta}{\gamma \sigma^{2}} e^{\frac{\beta^{2}}{\sigma^{2}} T}, \quad \forall t \in[0, T], \quad n=1,2, \ldots
$$

Step 2: Prove that $\left\{\dot{c}_{n}\right\}$ is uniformly bounded in $C[0, T]$.

From the defining recursion it is obvious that $c_{n}$ is continuously differentiable for all $n$, and we obtain

$$
\begin{aligned}
\dot{c}_{n}(t) & =\frac{\beta}{\gamma \sigma^{2}}\left[\left\{r+\beta c_{n-1}(t)+\sigma^{2} c_{n-1}^{2}(t)\right\} e^{-\int_{t}^{T}\left[r+\beta c_{n-1}(s)+\sigma^{2} c_{n-1}^{2}(s)\right] d s}\right. \\
& \left.+\gamma \sigma^{2} c_{n-1}(t) e^{-\int_{t}^{T} \sigma^{2} c_{n-1}^{2}(s) d s}\right] .
\end{aligned}
$$

Since $\left\{c_{n}\right\}$ is proved to be uniformly bounded, we conclude that $\left\{\dot{c}_{n}\right\}$ is uniformly bounded.

Step 3: Prove existence and uniqueness for $c$.

For any $s, t \in[0, T]$, and using the result from Step 2, we obtain

$$
\begin{aligned}
\left|c_{n}(t)-c_{n}(s)\right| & =\left|\int_{0}^{1} \frac{d}{d u} c_{n}(s+u(t-s)) d u\right| \\
& =\left|(t-s) \cdot \int_{0}^{1} \dot{c}_{n}(s+u(t-s)) d u\right| \leq K(t-s),
\end{aligned}
$$

where $K$ is a constant independent of $n$. Hence the sequence $\left\{c_{n}\right\}$ is equicontinuous and, since we have already proved uniform boundedness, the Arzela-Ascoli Theorem implies that there exists a $c \in C[0, T]$ and subsequence $\left\{c_{n_{i}}\right\}$ such that $c_{n_{i}} \rightarrow c$. Taking limits in (17) shows that $c$ is a solution to (13).

To prove uniqueness, assume that $c_{1}$ and $c_{2}$ are two solutions to (13). Noting that $c_{1}$ and $c_{2}$ are both bounded and that the function $\varphi(x)=e^{x}$ is globally Lipschitz on any given bounded set, we can show easily that

$$
\left|c_{1}(t)-c_{2}(t)\right| \leq K \int_{t}^{T}\left|c_{1}(s)-c_{2}(s)\right| d s .
$$

The Gronwal inequality now implies that $c_{1} \equiv c_{2}$.

Remark 4.2 The proof above shows that, for any subsequence of $\left\{c_{n}\right\}$ there is a further subsequence that converges to the same function $c$ (which is the solution to (13)). Thus $\left\{c_{n}\right\}$ itself converges to $c$. 


\subsection{A numerical algorithm for $c$}

The next question is to find a numerical algorithm for the determination of c. The obvious idea is of course to implement the recursion scheme (16)-(17) numerically. From Remark 4.2 we know that the scheme converges to $c$, and the following result gives the speed of convergence.

Theorem 4.3 Let $\left\{c_{n}\right\}$ be constructed according to (16)-(17), and let $c$ be the solution to (13). Then we have

$$
\left|c_{n}(t)-c(t)\right| \leq \sum_{m=n}^{\infty} \frac{K^{m-1}}{(m-2) !}(T-t)^{m-2}, \quad n=2,3, \ldots
$$

Proof. Let $\bar{c}_{n}=c_{n}-c_{n-1}$. Then, with arguments as in the proof above, it is easy to show that

$$
\left|\bar{c}_{n}(t)\right| \leq K \int_{t}^{T}\left|\bar{c}_{n-1}(s)\right| d s, \quad \forall t \in[0, T] .
$$

Denote $\varphi_{n}(t)=\int_{t}^{T}\left|\bar{c}_{n}(s)\right| d s$. From (18) we conclude that

$$
\dot{\varphi}_{n}(t)+K \varphi_{n-1}(t) \geq 0,
$$

which implies

$$
\varphi_{n}(t) \leq K \int_{t}^{T} \varphi_{n-1}(s) d s
$$

By induction we deduce

$$
\varphi_{n+1}(t) \leq \frac{K^{n}}{n !}(T-t)^{n} \varphi_{1}(0) .
$$

It then follows from (18) that

$$
\left|\bar{c}_{n}(t)\right| \leq K \varphi_{n-1}(t) \leq \frac{K^{n-1}}{(n-2) !}(T-t)^{n-2} \varphi_{1}(0),
$$

and the stated result follows.

\section{$5 \quad$ Numerical results}

We now illustrate the results for the case

$$
\gamma(x)=\frac{\gamma}{x}
$$

with various choices of $\gamma$, and for different time horizons. We have used the parameter values $\alpha=0.12, \sigma=0.2, r=0.04$. The function $c$, depicted in Figures 1 and 2, has been calculated using the iterative scheme (16)-(17). 
A natural comparison that one may make is between the mean-variance allocation problems and the standard Merton problem:

$$
\begin{array}{ll}
\max _{u} & E\left[U\left(t, X_{t}\right) d t\right] \\
& d X_{t}=\left[r X_{t}+(\alpha-r) u_{t}\right] d t+\sigma u_{t} d W_{t} .
\end{array}
$$

where $U(x)=\frac{x^{1-\gamma}}{1-\gamma}$. Figures 3-5 compare the investing strategies resulting from the two mean-variance problems and the standard Merton problem.

The Figure 3 plots the amount of money invested in the stock versus time as well as the proportion of wealth invested in the risky asset and the the risk aversion parameter versus time, computed for the three models. The Figure 4 plots the expected wealth (at the end of the investment horizon) versus time for the three problems, as well as the conditional variance of the final wealth. The Figure 5 presents plots the amount of money invested in the stock versus the wealth of the investor, as well as the proportion of money invested in the stock and the risk aversion parameter versus the wealth. This figure shows very similar the results yielded by the Merton model and the mean-variance with state dependent risk aversion. This is not surprising if one takes into account the fact that the risk aversion $\frac{\gamma}{x}$. However, the two models are not as similar when we look at how the optimal strategies behave along the timeline. In the Merton model, the proportion of wealth invested in the stock does not very with respect to the investment horizon:

$$
u(t, x)=\frac{\alpha-r}{\gamma \sigma^{2}} x .
$$

In the mean variance problem with state dependent risk aversion, the proportion of wealth invested in the stock decreases as the horizon increases. Also, the conditional variance of wealth is much lower in the case of the mean-variance problem with state-dependent risk aversion than in the other two approaches.

\section{A Proof of Proposition 4.1}

In order not to get swamped by details we write the wealth dynamics as

$$
d X_{t}=\mu\left(X_{t}, u_{t}\right) d t+\sigma\left(X_{t}, u_{t}\right) d W_{t},
$$

so we have, with obvious notation,

$$
\mathbf{A}^{u}=\frac{\partial}{\partial t}+\mu \frac{\partial}{\partial x}+\frac{1}{2} \sigma^{2} \frac{\partial^{2}}{\partial x^{2}}
$$

Denoting partial derivatives with subscript we now have

$$
\mathbf{A}^{u} V=V_{t}+\mu V_{x}+\frac{1}{2} \sigma^{2} V_{x x}
$$


Furthermore we have

$$
\mathbf{A}^{u} f^{x}(t, x)=f_{t}(t, x, x)+\mu f_{x}(t, x, x)+\frac{1}{2} \sigma^{2} f_{x x}(t, x, x)
$$

and

$$
\begin{aligned}
\mathbf{A}^{u} f(t, x, x)= & f_{t}(x, x)+\mu\left\{f_{x}(t, x, x)+f_{y}(t, x, x)\right\} \\
& +\frac{1}{2} \sigma^{2}\left\{f_{x x}(t, x, x)+f_{y y}(t, x, x)+2 f_{x y}(t, x, x)\right\} .
\end{aligned}
$$

Recalling that

$$
(G \diamond g)(t, x)=G(x, g(t, x))
$$

we also have

$$
\begin{aligned}
\mathbf{A}^{u}(G \diamond g)(t, x)= & G_{y} g_{t}+\mu G_{x}+\mu G_{y} g_{x} \\
& +\frac{1}{2} \sigma^{2}\left\{G_{x x}+G_{y} g_{x x}+2 G_{x y} g_{x}+G_{y y} g_{x}^{2}\right\} \\
= & G_{y} \mathbf{A}^{u} g \\
& +\mu G_{x}+\frac{1}{2} \sigma^{2}\left\{G_{x x}+2 G_{x y} g_{x}+G_{y y} g_{x}^{2}\right\}
\end{aligned}
$$

where $G$ is evaluated at $G(x, g(t, x))$ and $g$ is evaluated at $g(t, x)$.

Since

$$
\mathbf{H}^{u} g(t, x)=G_{y}(x, g(t, x)) \cdot \mathbf{A}^{u} g(t, x)
$$

we see that the extended HJB system takes the form

$$
\begin{aligned}
V_{t}+\sup _{u \in \mathcal{U}}\left\{\mu V_{x}+\frac{1}{2} \sigma^{2} V_{x x}-\mu f_{y}-\frac{1}{2} \sigma^{2} f_{y y}-\sigma^{2} f_{x y}\right. & \\
\left.-\mu G_{x}-\frac{1}{2} \sigma^{2}\left[G_{x x}+2 G_{x y} g_{x}+G_{y y} g_{x}^{2}\right]\right\} & =0, \\
f_{t}(t, x, y)+\hat{\mu} f_{x}(t, x, y)+\frac{1}{2} \hat{\sigma}^{2} f_{x x}(t, x, y) & =0, \\
g_{t}(t, x)+\hat{\mu} g_{x}(t, x)+\frac{1}{2} \hat{\sigma}^{2} g_{x x}(t, x) & =0 .
\end{aligned}
$$

Note that in (19), the function $f$ and its derivatives should be evaluated as $f(t, x, x)$ and similarly for $f_{t}, f_{x}$, and $f_{x x}$. In (20)-(21) we have used the shorthand notation

$$
\begin{aligned}
& \hat{\mu}=\mu(x, \hat{u}(t, x)), \\
& \hat{\sigma}=\sigma(x, \hat{u}(t, x)) .
\end{aligned}
$$

We recall that in our case we have

$$
\begin{aligned}
\mu(x, u) & =r x+(\alpha-r) u \\
\sigma(x, u) & =\sigma u .
\end{aligned}
$$


Using the notation

$$
\beta=\alpha-r
$$

the extended HJB system is thus as follows,

$$
\begin{aligned}
V_{t}+\sup _{u \in R}\left\{(r x+\beta u)\left[V_{x}-f_{y}-G_{x}\right]\right. & \\
\left.+\frac{1}{2} \sigma^{2} u^{2}\left[V_{x x}-f_{y y}-2 f_{x y}-G_{x x}-2 G_{x y} g_{x}-G_{y y} g_{x}^{2}\right]\right\} & =0, \\
f_{t}(t, x, y)+(r x+\beta \hat{u}) f_{x}(t, x, y)+\frac{1}{2} \sigma^{2} \hat{u}^{2} f_{x x}(t, x, y) & =0, \\
g_{t}(t, x)+(r x+\beta \hat{u}) g_{x}(t, x)+\frac{1}{2} \sigma^{2} \hat{u}^{2} g_{x x}(t, x) & =0 .
\end{aligned}
$$

Note the following

- In (22) the function $f_{t}$ and other $f$-derivatives should be evaluated as $f_{t}(t, x, x)$ etc.

- In (22), the function $G_{x}$ and other derivatives should be evaluated as $G_{x}(x, g(t, x))$ etc.

For completeness we also recall the dynamics

$$
d X_{t}=\left[r X_{t}+\beta u_{t}\right] d t+\sigma u_{t} d W_{t}
$$

Furthermore we have

$$
\begin{aligned}
G_{x}(x, y) & =\frac{\gamma^{\prime}(x)}{2} y^{2}, \\
G_{x x}(x, y) & =\frac{\gamma^{\prime \prime}(x)}{2} y^{2} \\
G_{y}(x, y) & =\gamma(x) y \\
G_{x y}(x, y) & =\gamma^{\prime}(x) y \\
G_{y y}(x, y) & =\gamma(x) .
\end{aligned}
$$

Plugging these expressions for the $G$ derivatives into the extended HJB proves Proposition 4.1.

\section{B Proof of Proposition 4.3}

In this special case we have

$$
\begin{aligned}
\gamma(x) & =\frac{\gamma}{x}, \\
\gamma^{\prime}(x) & =-\frac{\gamma}{x^{2}}, \\
\gamma^{\prime \prime}(x) & =\frac{2 \gamma}{x^{3}} .
\end{aligned}
$$


We now conjecture that $\hat{u}$ is linear in $x$ so we make the Ansatz

$$
\hat{u}(t, x)=c(t) x
$$

for some deterministic function $c$. If this is the case, then $X$ will be GBM so we will have

$$
\begin{aligned}
E_{t, x}\left[X_{T}^{\hat{\mathbf{u}}}\right] & =a(t) x, \\
E_{t, x}\left[\left(X_{T}^{\hat{\mathbf{u}}}\right)^{2}\right] & =b(t) x^{2},
\end{aligned}
$$

for some deterministic functions $a$ and $b$. We recall the probabilistic interpretations

$$
\begin{aligned}
f(t, x, y) & =E_{t, x}\left[X_{T}^{\hat{\hat{u}}}\right]-\frac{\gamma(y)}{2} E_{t, x}\left[\left(X_{T}^{\hat{\hat{u}}}\right)^{2}\right], \\
g(t, x) & =E_{t, x}\left[X_{T}^{\hat{\hat{u}}}\right] .
\end{aligned}
$$

This leads to the Ansatz

$$
\begin{aligned}
f(t, x, y) & =a(t) x-\frac{\gamma}{2 y} b(t) x^{2}, \\
g(t, x) & =a(t) x .
\end{aligned}
$$

With this we have, with $\dot{a}=\frac{\partial a}{\partial t}$ etc,

$$
\begin{aligned}
f_{t}(t, x, y) & =\dot{a} x-\frac{\gamma}{2 y} \dot{b} x^{2}, & f_{x}(t, x, y) & =a-\frac{\gamma}{y} b x \\
f_{x x}(t, x, y) & =-\frac{\gamma}{y} b, & f_{x y}(t, x, y) & =\frac{\gamma}{y^{2}} b x \\
f_{y}(t, x, y) & =\frac{\gamma}{2 y^{2}} b x^{2}, & f_{y y}(t, x, y) & =-\frac{\gamma}{y^{3}} b x^{2} \\
g_{t}(t, x) & =\dot{a} x, & g_{x}(t, x) & =a \\
g_{x x}(t, x) & =0 . & &
\end{aligned}
$$

Plugging this into (9) gives us the equilibrium control as

$$
\hat{u}(t, x)=\frac{\beta}{\gamma \sigma^{2} b}\left(a+\gamma\left[a^{2}-b\right]\right) x .
$$

Equation (10) now reads

$$
\begin{aligned}
& \dot{a} x-\frac{\gamma}{2 y} \dot{b} x^{2}+\left(r x+\frac{\beta^{2}}{\gamma \sigma^{2} b}\left(a+\gamma\left[a^{2}-b\right]\right) x\right)\left(a-\frac{\gamma}{y} b x\right) \\
& -\frac{1}{2} \frac{\beta^{2}}{\gamma^{2} \sigma^{2} b^{2}}\left(a+\gamma\left[a^{2}-b\right]\right)^{2} x^{2} \frac{\gamma}{y} b=0 .
\end{aligned}
$$


By separation of variables this leads to the following system of ODEs

$$
\begin{aligned}
\dot{a}+\left(r+\frac{\beta^{2}}{\gamma \sigma^{2} b}\left(a+\gamma\left[a^{2}-b\right]\right)\right) a & =0, \\
\dot{b}+\left\{2\left(r+\frac{\beta^{2}}{\gamma \sigma^{2} b}\left(a+\gamma\left[a^{2}-b\right]\right)\right)+\frac{\beta^{2}}{\gamma^{2} \sigma^{2} b^{2}}\left(a+\gamma\left[a^{2}-b\right]\right)^{2}\right\} b & =0 .
\end{aligned}
$$

Equation (11) becomes

$$
\dot{a} x+\left(r x+\frac{\beta^{2}}{\gamma \sigma^{2} b}\left(a+\gamma\left[a^{2}-b\right]\right) x\right) a=0,
$$

leading to the ODE

$$
\dot{a}+\left(r+\frac{\beta^{2}}{\gamma \sigma^{2} b}\left(a+\gamma\left[a^{2}-b\right]\right)\right) a=0,
$$

which we recognize from above.

\section{References}

[1] Bajeux-Besnainou, I., And Portait, R. Dynamic asset allocation in a mean-variance framework. Management Science 44, 11 (1998), 79-95.

[2] Basak, S., and Chabakauri, G. Dynamic mean-variance asset allocation. Review of Financial Studies 23 (2010), 2970-3016.

[3] Bielecki, T. R., Jin, H., Pliska, S., And Zhou, X. Continuous-time mean-variance portfolio selection with bankruptcy prohibition. Mathematical Finance 15, 2 (2005), 213-244.

[4] Björk, T., And Murgoci, A. A general theory of Markovian time inconsistent stochastic control problems. Working paper, Stockholm School of Economics, 2009.

[5] Dai, M., Xu, Z., and Zhou, X. Continuous-time Markowitz's model with transaction costs. SIAM Journal on Financial Mathematics 1 (2010), 96-125.

[6] Ekeland, I., AND LazraK, A. Being serious about non-commitment: subgame perfect equilibrium in continuous time, 2006. Preprint. University of British Columbia.

[7] Ekeland, I., And Privu, T. Investment and consumption without commitment, 2007. Preprint. University of British Columbia.

[8] Goldman, S. Consistent plans. Review of Economic Studies 47 (1980), $533-537$. 
[9] Krusell, P., And Smith, A. Consumption and savings decisions with quasi-geometric discounting. Econometrica 71 (2003), 366-375.

[10] Kydland, F. E., And Prescott, E. Rules rather than discretion: The inconsistency of optimal plans. Journal of Political Economy 85 (1997), 473-492.

[11] LI, D., AND NG, W. Optimal dynamic portfolio selection: Multi-period mean-variance formulation. Mathematical Finance 10 (2000), 387-406.

[12] Lim, A. E. B. Quadratic hedging and mean-variance portfolio selection with random parameters in an incomplete market. Mathematics of Operations Research 29 (2004), 132-161.

[13] Lim, A. E. B., AND Zhou, X. Quadratic hedging and mean-variance portfolio selection with random parameters in a complete market. Mathematics of Operations Research 27, 1 (2002), 101-120.

[14] Markowitz, H. Portfolio selection. Journal of Finance 7 (1952), 77-98.

[15] Peleg, B., And Menahem, E. On the existence of a consistent course of action when tastes are changing. Review of Economic Studies 40 (1973), 391-401.

[16] Pollak, R. Consistent planning. Review of Economic Studies 35 (1968), 185-199.

[17] Richardson, H. R. A minimum variance result in continuous trading portfolio optimization. Management Science 35, 9 (1989), 1045-1055.

[18] Strotz, R. Myopia and inconsistency in dynamic utility maximization. Review of Economic Studies 23 (1955), 165-180.

[19] Vieille, N., AND Weibull, J. Multiple solutions under quasi-exponential discounting. Economic Theory 39 (2009), 513-526.

[20] XIA, J. M. Mean-variance portfolio choice: Quadratic partial hedging. Mathematical Finance 15, 3 (2005), 533-538.

[21] Zhou, X. Y., AND Li, D. Continuous-time mean-variance portfolio selection: A stochastic lq framework. Applied Mathematics and Optimization 42 (2000), 19-33. 

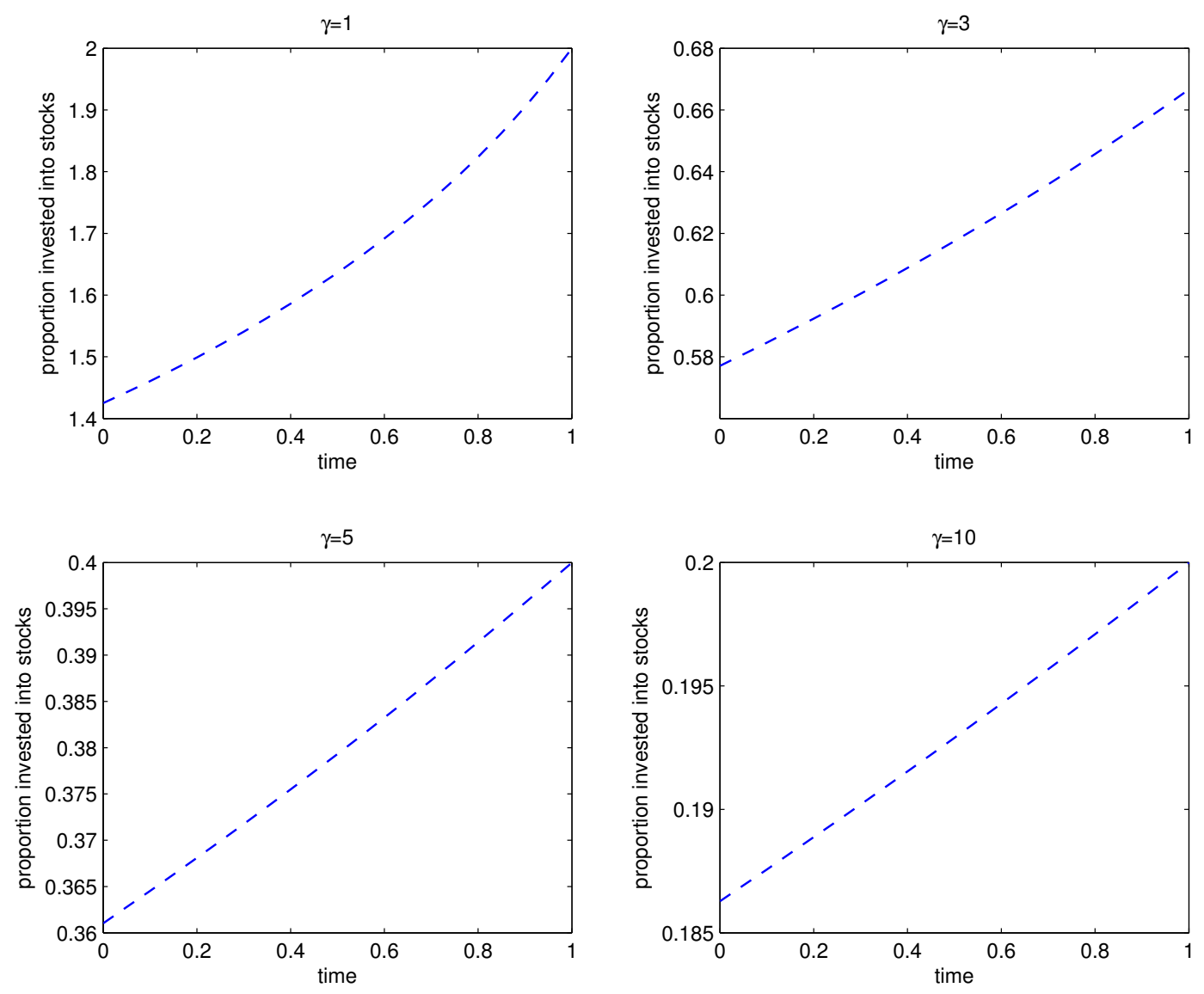

Figure 1: The function $c(t)$ for various choices of $\gamma$ with $T=1$. 

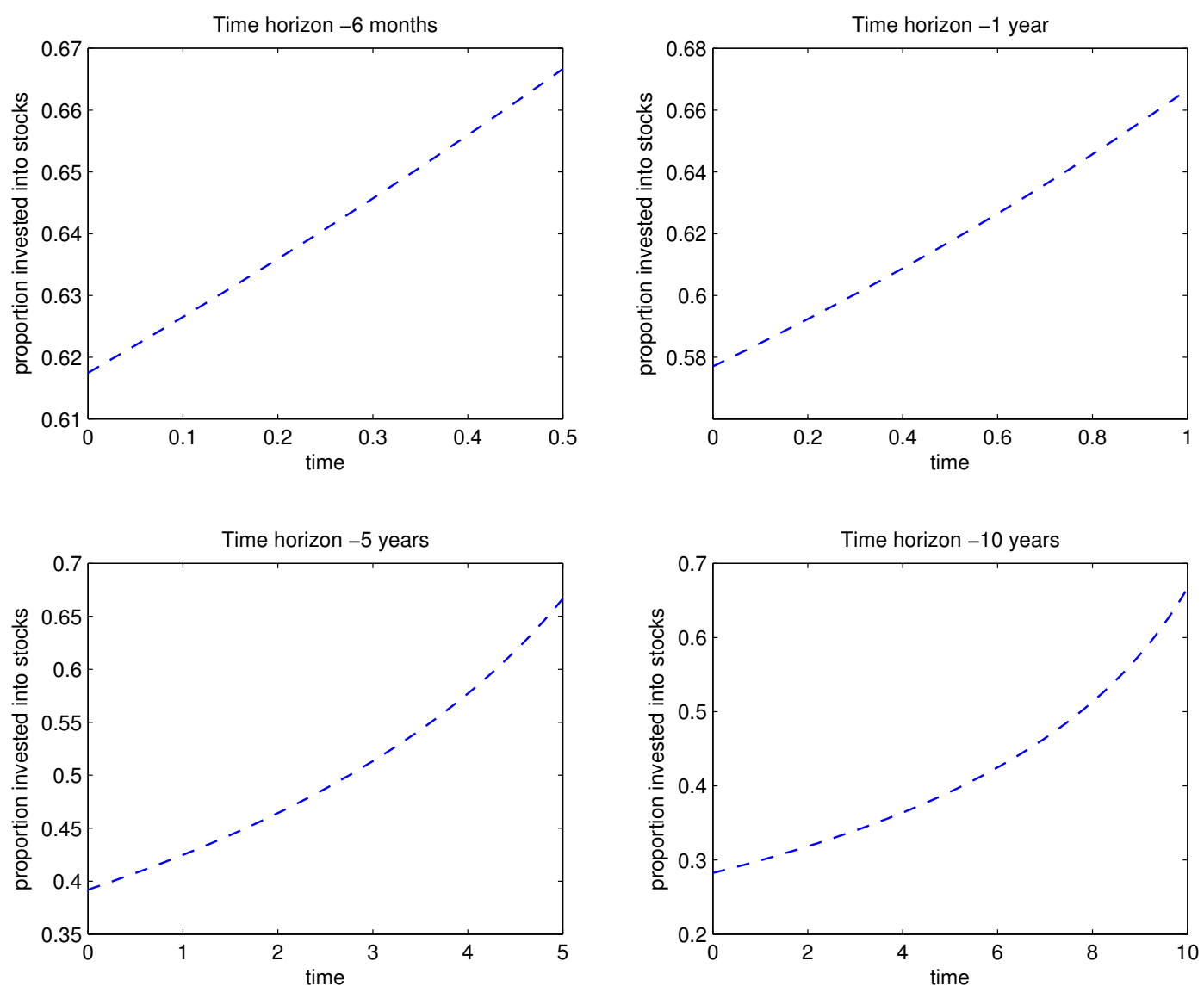

Figure 2: The function $c(t)$ for various time horizons with $\gamma=3$. 

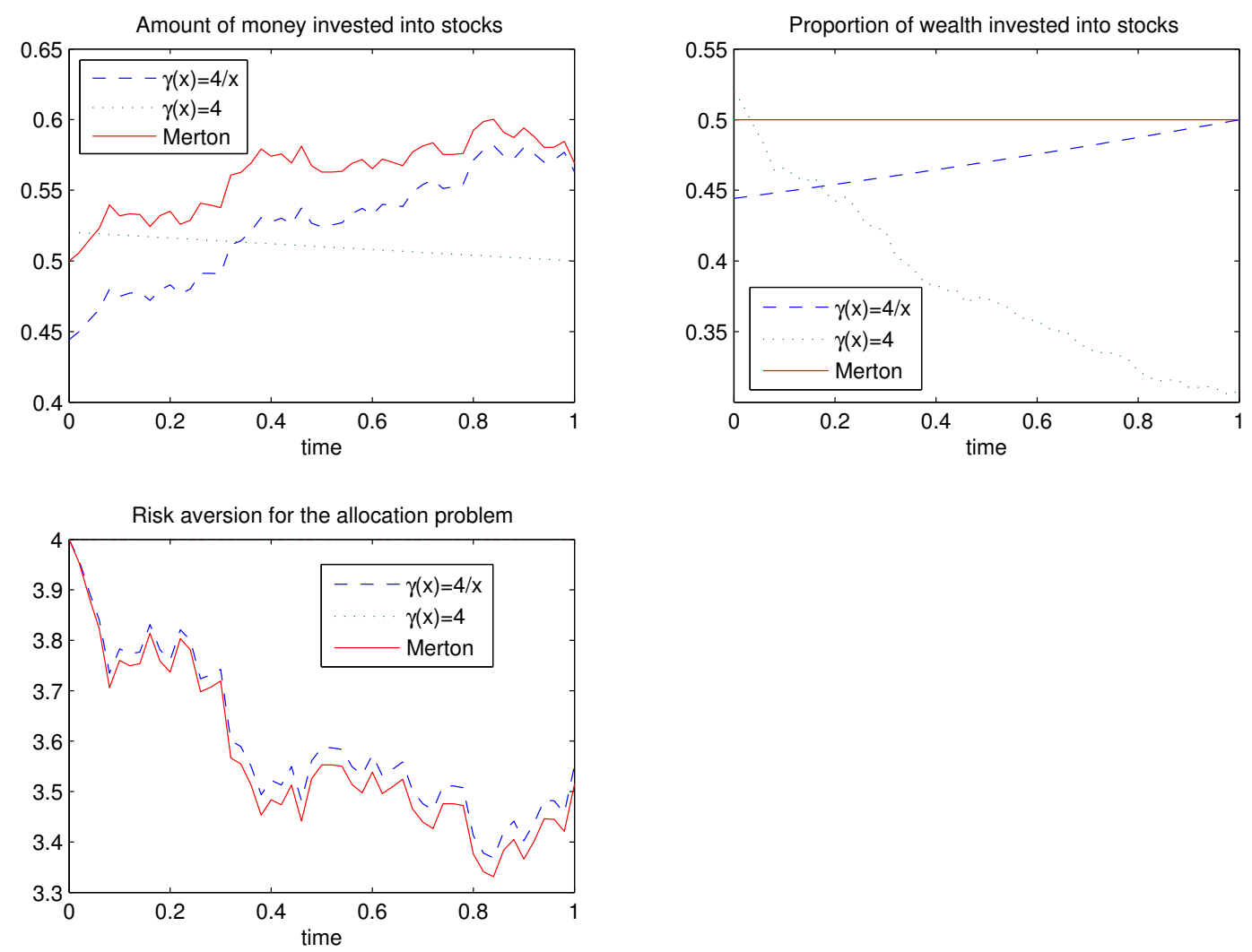

Figure 3: 

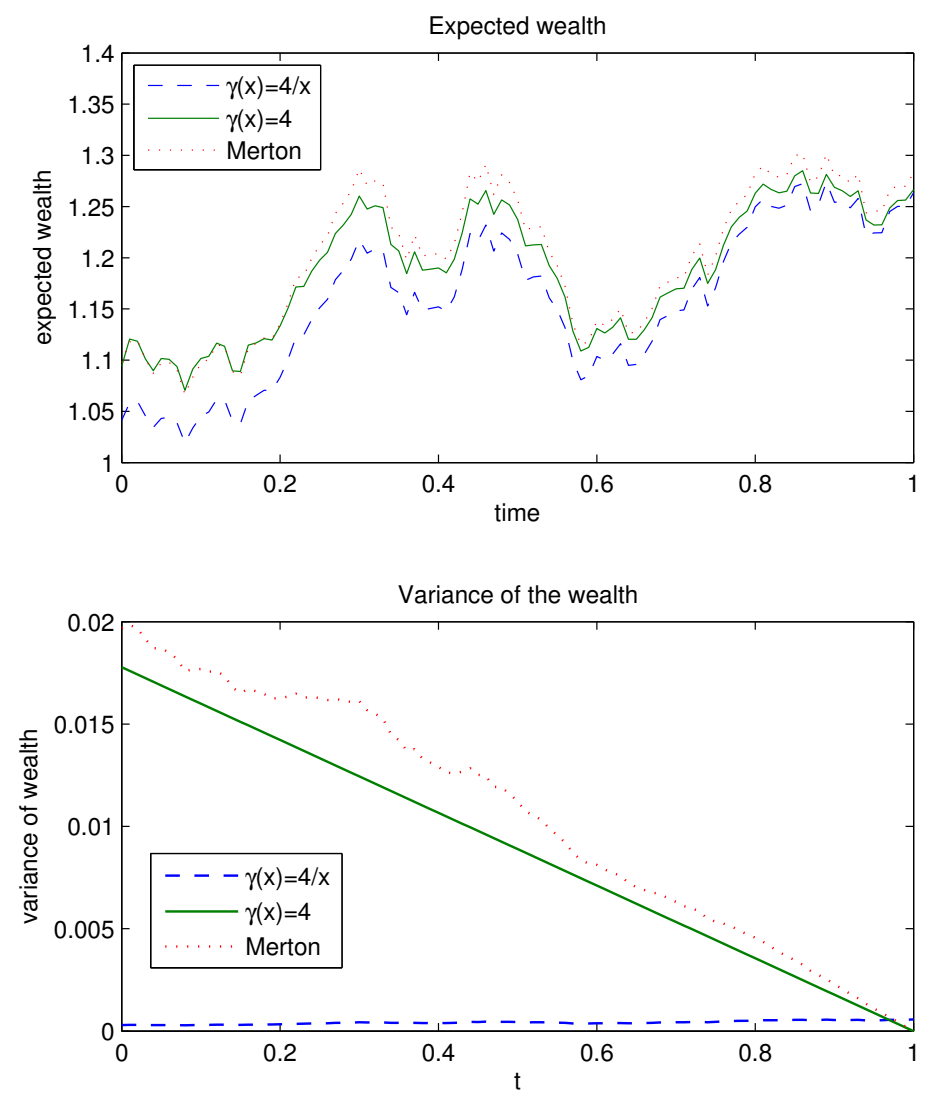

Figure 4: Expected wealth and the conditional variance of wealth in the three models 

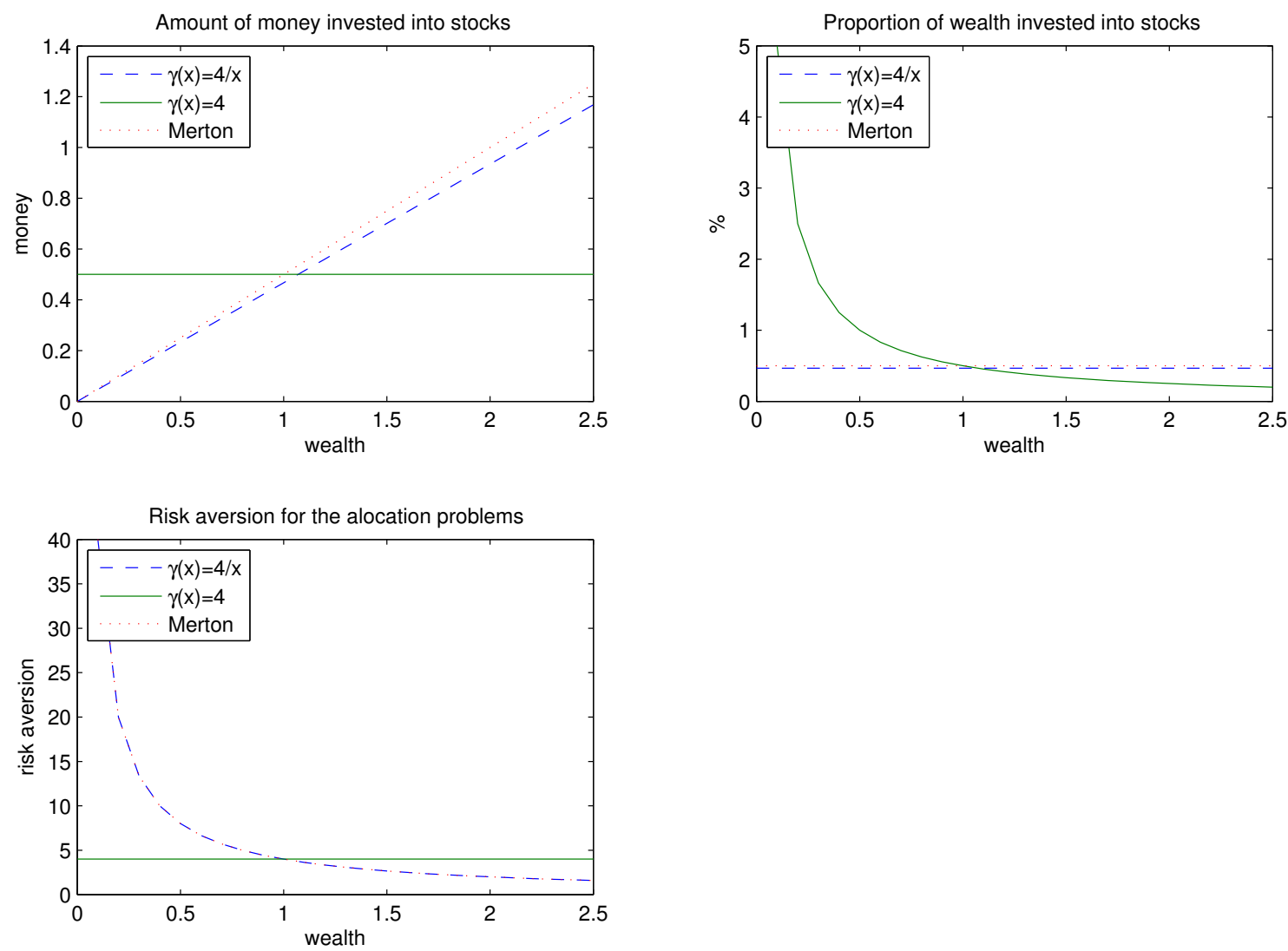

Figure 5: Amount of money invested in the stock, proportion of money invested in the stock and risk aversion across wealth levels in the three models 